\title{
Petrous Apex Cholesterol Granuloma: Importance of Pedicled Nasoseptal Flap in Addition to Silicone T-tube for Prevention of Occlusion of Drainage Route in Transsphenoidal Approach-A Technical Note
}

\author{
Shunsuke ShibaO, ${ }^{1}$ Masahiro TodA, ${ }^{1}$ Toshiki Tomita, ${ }^{2}$ Katsuya SAIto, ${ }^{1}$ \\ Kaoru OGAWA, ${ }^{2}$ Takeshi KaWASE, ${ }^{1}$ and Kazunari YOSHIDA ${ }^{1}$ \\ Departments of ${ }^{1}$ Neurosurgery and ${ }^{2}$ Otorhinolaryngology, Head, and Neck Surgery, \\ Keio University Graduate School of Medicine, Tokyo
}

\begin{abstract}
Recently, petrous apex cholesterol granulomas (CGs) have been treated via the endoscopic endonasal transsphenoidal approach (EEA) using a silicone tube, to prevent drainage route occlusion. Occlusion of the drainage route has led to problems with recurrence. The aim of this report is to describe the use of a surgical technique to prevent drainage route occlusion. In surgical technique, the posterolateral wall of the sphenoid sinus was opened by EEA. After cyst debridement, a vascularized nasoseptal flap with a width of approximately $4 \mathrm{~cm}$ was inserted into the lumen with a silicone T-tube with a diameter of $7 \mathrm{~mm}$. This technique was used in two patients: the first patient during the second operation after recurrence following occlusion of the drainage route, and the second patient during the first operation. Opening of the cyst wall was confirmed endoscopically in both patients 12-24 months after surgery, even after removal of the T-tube. In conclusion, the use of a pedicled nasoseptal flap with a silicone tube is useful to prevent CG recurrence, by paranasal cavitization of the cystic cavity.
\end{abstract}

Key words: cholesterol granuloma, pedicled nasoseptal flap, petrous apex, silicone tube, transsphenoidal approach

\section{Introduction}

Cholesterol granuloma (CG) of the petrous apex arises due to an inflammatory reaction to the byproducts of eroded marrow cavities secondary to chronic obstruction of air cells within the petrous apex. ${ }^{1,2)}$ Various approaches have been used for the drainage and permanent aeration of the petrous apex, such as the transsphenoidal approach, middle fossa approach, and transpetrous approach, with the aim of preventing CG recurrence. However, the recurrence rate of petrous apex CG remains high, at up to $60 \%{ }^{3)}$

The endoscopic endonasal approach (EEA) has recently been developed as a minimally invasive surgery for petrous apex CGs. In fact, 11 cases of EEA to treat petrous apex CGs have been reported to date, in which a silicone tube was used to prevent drainage route occlusion. Occlusion of the drainage route has led to problems with recurrence. Here, we report a surgical technique that can be used to

Received July 22, 2014; Accepted October 13, 2014 prevent drainage route occlusion: EEA using a pedicled nasoseptal flap in addition to a silicone T-tube, for more reliable patency of the drainage route.

\section{Surgical Technique}

After entering the nasal cavity and resecting the middle turbinate, we made a vascularized nasoseptal flap measuring approximately $4 \mathrm{~cm}$ wide and resected the anterior wall of the sphenoid sinus. Then, the posterolateral wall of the sphenoid sinus was opened and cyst debridement was performed. After that we overlaid the vascularized nasoseptal flap into the drainage lumen with a silicone T-tube measuring $7 \mathrm{~mm}$ in diameter.

\section{Results}

\section{Case 1}

A 36-year-old woman presented with vertigo, left otorrhea, and left hearing disturbance, which she had experienced for 3 years, 1 year, and 8 months, 
respectively. She had no history of head trauma or surgery. Although she denied any recent history of otitis media, she reported occasional episodes of this condition during high school. These episodes were treated medically with no complications. A neurological examination revealed left conductive hearing impairment; no other cranial nerve deficits were detected.

Computed tomography (CT) of the petrous bone and skull base performed on admission showed an expansile mass in the left petrous apex with bone remodeling of the clivus and skull base (Fig. 1A). Magnetic resonance imaging (MRI) revealed a large left petrous apex mass abutting the sphenoid sinus (Fig. 1B). The preoperative diagnosis was CG. The patient was referred to our institution from another medical center for surgery via a transcranial approach, such as the anterior transpetrosal approach; however, an EEA was planned to achieve permanent drainage.

Endoscopic drainage of the granuloma was performed through the right naris. The middle turbinate was resected, and a wide sphenoidotomy was performed, providing access to a large sinus. The inferior posterolateral wall of the sphenoid sinus, i.e., the clivus,

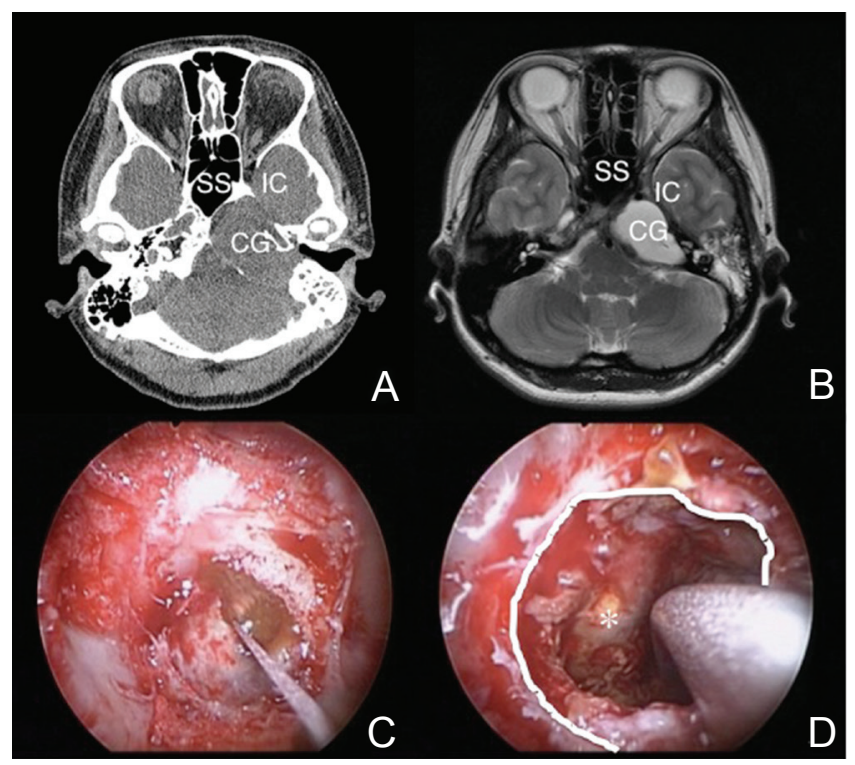

Fig. 1 Case 1, first operation. A: Computed tomography (CT) scan shows bone erosion caused by expansile mass in the left petrous apex. $B: T_{2}$-weighted magnetic resonance imaging (MRI) reveals a hyperintense mass in the left petrous apex. C: After removing the inferior posterolateral wall of the sphenoid sinus and cutting the cyst wall, a brownish fluid, typical of CG, flowed out. D: After debridement of the granuloma, the dura of the posterior fossa (asterisk) was identified through the fenestration (solid line). CG: cholesterol granuloma, IC: internal carotid artery, SS: sphenoid sinus. was removed, revealing a large, dark, fluid-filled cyst in the petrous apex (Fig. 1C). The cyst was opened, drained, and widely marsupialized, and the dura of the posterior fossa was identified through the fenestration, measuring $15 \mathrm{~mm}$ in diameter (Fig. 1D). A drainage tube was not inserted because of the wide fenestration. The postoperative course was uneventful and the symptoms of vertigo, otorrhea, and hearing disturbance improved.

Two months after the operation, however, fiberscopic examination revealed reocclusion of the cyst fenestration. MRI revealed a scar occluded at the fenestration site and reaccumulation of fluid in the cyst (Fig. 2A). The patient underwent a second endoscopic operation, through the right naris, for the placement of a drainage tube with a pedicled nasoseptal flap. The drainage port was obstructed by thick granulation tissue adjacent to

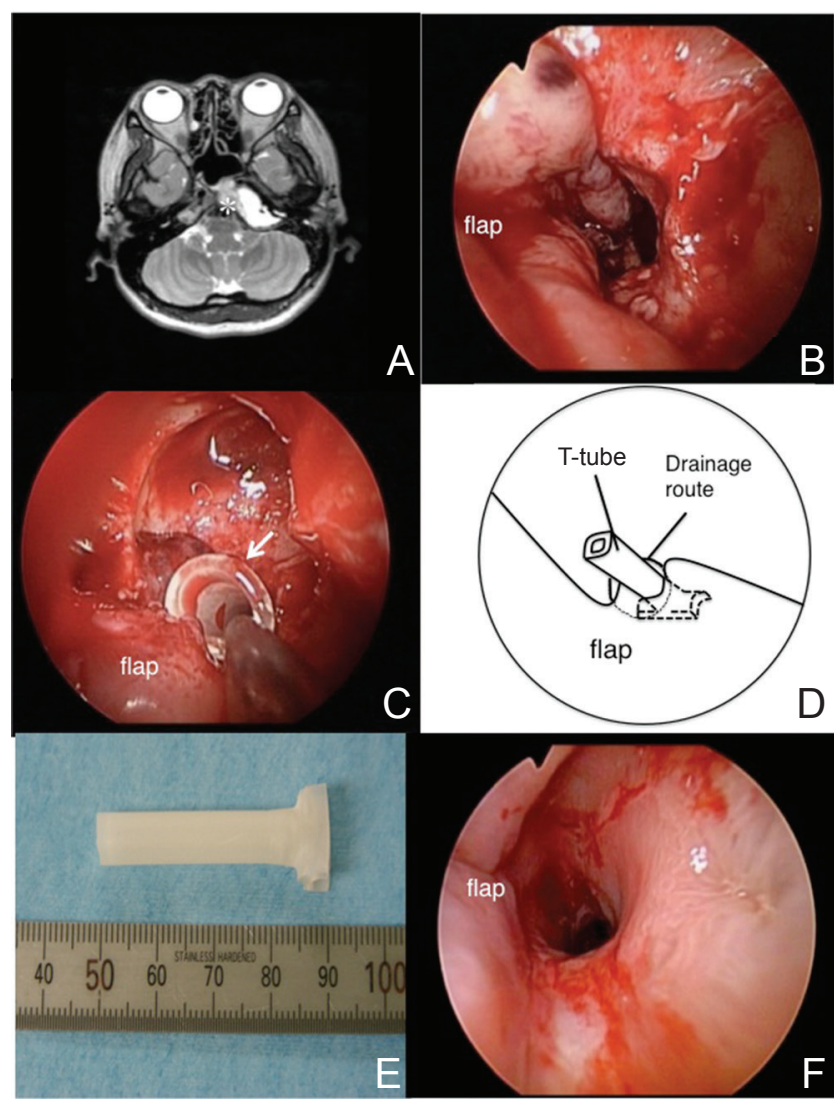

Fig. 2 Case 1, second operation. A: Preoperative $\mathrm{T}_{2}$-weighted magnetic resonance imaging (MRI) reveals obstruction by a scar (asterisk) at the fenestration site and reaccumulation of fluid in the cyst. The nasoseptal flap was partially overlaid (B), and a modified silicone T-tube (arrow) was placed in the drainage port (C). D, E: The schema and the T-tube. F: Two years after surgery, patent cyst fenestration was confirmed by fiberscopic examination after T-tube removal. 
the left internal carotid artery (ICA). The granulation tissue was removed after checking the location of the ICA using a navigation system (StealthStation, Medtronic, Inc., Tokyo), and the cyst was reopened. To prevent recurrence, a vascularized nasoseptal flap was elevated and overlaid around the drainage port. A modified silicone T-tube (RD-T drain, CREATE MEDIC Co., Yokohama, Kanagawa) was cut to $4 \mathrm{~cm}$ in length and placed into the cyst cavity with the flap (Fig. 2B-E). The postoperative course after the second operation was uneventful. Two years later, the T-tube was removed, and fiberscopic examination showed that the orifice of the cyst was preserved and covered with mucosa (Fig. 2F).

\section{Case 2}

A 34-year-old woman visited a nearby hospital for headache. She had no otological complaint, and neurological examination was normal. MRI of the head demonstrated a large lesion $(36 \times 22 \mathrm{~mm})$ with high signal on $\mathrm{T}_{1^{-}}$and $\mathrm{T}_{2}$-weighted images in the left petrous apex, expanding into the posterior fossa (Fig. 3A). There was evidence of bony erosion on CT scan. Imaging also showed right maxillary sinusitis. She was referred to our department for EEA, and we planned the same procedure as in Case 1 for this patient.

The lesion was approached by EEA using an image guidance system (StealthStation, Medtronic, Inc.). A $1.5-\mathrm{cm}$ stoma was created to allow the drainage of the CG in the petrous apex (Fig. 3B). After complete debridement of the cyst, a vascularized nasoseptal flap was elevated and overlaid around the drainage route (Fig. 3C). A modified silicone T-tube (RD-T drain, CREATE MEDIC Co.) was cut to $3 \mathrm{~cm}$ in length and placed with the flap to secure the drainage route (Fig. 3D). The patient recovered uneventfully and was discharged 7 days after the procedure. Her headache resolved completely within the first week of the postoperative period. The diagnosis of CG was confirmed histologically and the T-tube was removed 6 months after surgery. Fiberscopic examination demonstrated complete opening of the cyst, and the orifice was covered with the nasoseptal flap (Fig. 3E). At the 1-year follow-up, MRI showed no recurrence (Fig. 3F) and the patient reported significant improvement of her headache.

\section{Discussion}

In 1983, a CG was first reported in the peritoneum. ${ }^{4)}$ In 1947, Waltner and Karatay described a large CG involving the mastoid cavity and petrous apex.5) Subsequently, various authors reported cholesterol cysts of the petrous apex as a distinct clinical entity. ${ }^{6,7)}$ CGs of the petrous apex are uncommon because a minority of temporal bones (30\%) show pneumatization of the petrous apex. ${ }^{8)}$

The treatment of symptomatic petrous apex CGs is a surgical drainage and permanent aeration, to prevent recurrence. ${ }^{9)}$ Various surgical approaches, including the transsphenoidal approach, middle cranial fossa approach, and lateral-transtemporal approach, have been used for the drainage of petrous apex CGs. The transpetrous approach includes infralabyrinthine, infracochlear, transcochlear, and translabyrinthine approaches. ${ }^{10)}$ The transsphenoidal approach was first described

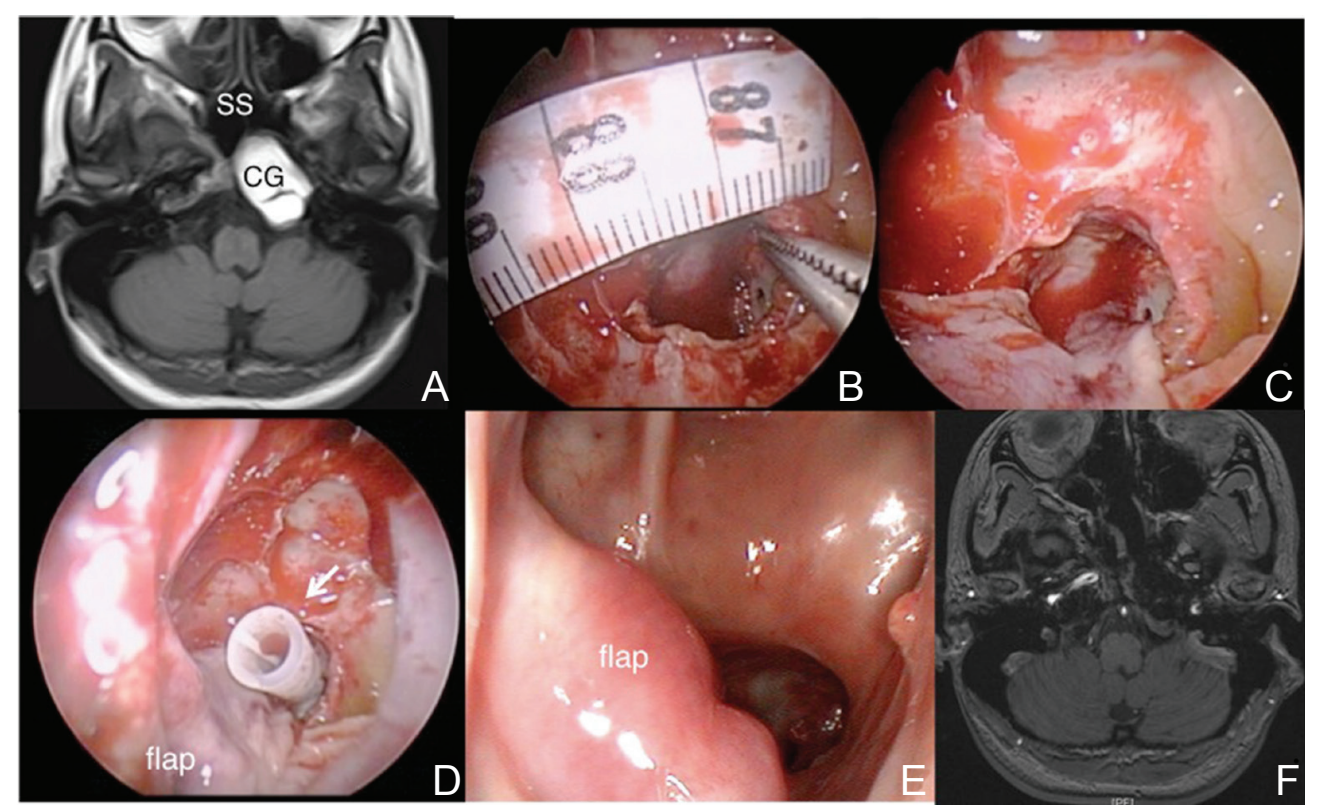

Fig. 3 Case 2. A: $\mathrm{T}_{1}$-weighted magnetic resonance imaging (MRI) reveals a hyperintense mass in the left petrous apex. B: A stoma created to allow drainage of the CG in the petrous apex. The septal flap was partially overlaid (C) and a modified silicone T-tube (arrow) was placed in the drainage port (D). E: Patent cyst fenestration was confirmed after T-tube removal by fiberscopic examination 1 year after surgery. $\mathrm{F}$ : $\mathrm{T}_{1}$-weighted MRI reveals no recurrence of the lesion in the left petrous apex. CG: cholesterol granuloma, SS: sphenoid sinus. 
by Montgomery in 1977, and the EEA was first reported by Fucci et al. in 1994. ${ }^{11,12)}$

The transsphenoidal approach has several advantages: it is technically less challenging and leads to better preservation of the function of the acoustic and facial nerves compared with other approaches. Patients treated by the transsphenoidal approach can be followed on an outpatient basis using a fiberscope and, where necessary, drainage of the cyst can be re-established. ${ }^{13)}$ In addition, this approach can be used to create large drainage routes. However, the approach is hampered by the complex anatomical structure, with limited indication in a patient showing sufficient drainage space medial to the ICA, and inferior to the pituitary gland. Therefore, the approach should be used with a navigation system to check the locations of critical neighboring structures. $^{14)}$

Some cases of EEA for the treatment of petrous apex CGs have been reported since Fucci et al. first reported the technique in 1994. We reviewed 13 cases of petrous apex CGs treated by EEA with regard to the technique and drainage route occlusion (Table 1). ${ }^{8-11,13,15,16,19)}$ Silicone tubes were placed in two cases, and the sphenoid mucosa was preserved in one case, to prevent occlusion of the drainage route. $^{9-11)}$ In these cases, the drainage route was not occluded. However, in 10 patients, in whom the drainage route was not secured using a silicone tube or mucosal flap, $5(50 \%)$ presented reaccumulation by occlusion ( 4 cases) or stenosis (1 case) of the drainage route. ${ }^{8,10,15,16)}$ The time until occlusion or stenosis ranged from 3 months to 2 years. This implies that a silicone tube or mucosal covering can prevent occlusion of the drainage route. In Case 1 , solely opening the drainage route resulted in occlusion after the first operation. The time until occlusion was 2 months, which is the shortest period reported to date. Therefore, a vascularized nasoseptal flap was overlaid to prevent scarring and subsequent occlusion, and a modified silicone T-tube was used to secure the drainage stoma. Because drainage routes become occluded at high rates despite wide fenestration, they should be covered with a drainage tube and/or vascularized mucosa, if possible.

The detailed description of the combination of a silicone tube and a vascularized nasoseptal flap has not been previously reported, to the best of our knowledge. The benefit of this combination is the more reliable prevention of drainage route occlusion because a vascularized flap could prevent granulation at the drainage site. The usefulness of a vascularized flap in the middle fossa approach was reported by Kusumi et al. ${ }^{17)}$ Paluzzi et al. reported the mucoperiosteal miniflap in 4 cases of 17 petrous apex CGs, and permanent aeration was achieved. ${ }^{18)}$ Furthermore, we used a T-shaped silicone tube, rather than a straight silicone tube. The T-tube has the advantage of hooking on its horizontal part and preventing the tube from dropping out of the cavity by gravity. The period until tube removal is

Table 1 Features of petrous apex cholesterol granulomas reported in literature and in the present case

\begin{tabular}{|c|c|c|c|c|c|}
\hline & $\begin{array}{c}\text { Age } \\
\text { (years) }\end{array}$ & Sex & Intervention & $\begin{array}{l}\text { Reocclusion (O) } \\
\text { or restenosis (S) }\end{array}$ & $\begin{array}{l}\text { Time until } \\
\text { reocclusion or restenosis }\end{array}$ \\
\hline Fucci et al. (1994) ${ }^{11)}$ & 36 & $\mathrm{~F}$ & Tube & - & - \\
\hline \multirow[t]{2}{*}{ Griffith and Terrell (1996) ${ }^{8)}$} & 34 & M & - & $\mathrm{O}$ & 3 months \\
\hline & 24 & M & - & $\mathrm{O}$ & 3 months \\
\hline Michaelson et al. (2001) & 13 & $\mathrm{~F}$ & - & - & - \\
\hline DiNardo et al. (2003) ${ }^{15)}$ & 62 & $\mathrm{~F}$ & - & $\mathrm{S}$ & 12 months \\
\hline Oyama et al. $(2007)^{13)}$ & 28 & $\mathrm{~F}$ & - & - & - \\
\hline \multirow[t]{4}{*}{ Georgalas and Kania (2008) ${ }^{10)}$} & 30 & M & - & $\mathrm{O}$ & Not reported \\
\hline & 13 & M & Mucosa & - & - \\
\hline & 34 & $\mathrm{~F}$ & - & $\mathrm{O}$ & 2 years \\
\hline & 58 & M & - & - & - \\
\hline Samadian et al. (2009) ${ }^{9)}$ & 28 & $\mathrm{~F}$ & Tube & - & - \\
\hline \multirow[t]{2}{*}{ Jaberoo et al. $(2010)^{19)}$} & 53 & M & - & - & - \\
\hline & 32 & $\mathrm{~F}$ & - & - & - \\
\hline Present case & 36 & $\mathrm{~F}$ & - & $\mathrm{O}$ & 2 months \\
\hline
\end{tabular}

F: female, M: male. 
determined based on mucosa covering confirmed by fiberscopic examination. In our cases, the period ranged from 6 months to 2 years.

\section{Conclusion}

We have described a surgical technique that can be used to prevent drainage route occlusion, and applied this technique to two cases of petrous apex CG. The use of a pedicled nasoseptal flap in addition to a silicone tube is useful to prevent occlusion of the cyst, by paranasal cavitization of the cystic cavity.

\section{Conflicts of Interest Disclosure}

The authors have no personal or institutional financial interest in any of the drugs, materials, or devices described in this article.

\section{References}

1) Farrior B, Kampsen E, Farrior JB: The positive pressure of cholesterol granuloma idiopathic blue eardrum. differential diagnosis. Laryngoscope 91: 1286-1297, 1981

2) Sheehy JL, Linthicum FH, Greenfield EC: Chronic serous mastoiditis, idiopathic hemotympanum and cholesterol granuloma of the mastoid. Laryngoscope 79: 1189-1217, 1969

3) Eisenberg MB, Haddad G, Al-Mefty O: Petrous apex cholesterol granulomas: evolution and management. J Neurosurg 86: 822-829, 1997

4) Meyer C: Fremdkorperperitonitis mit Bildung von riesenzellhaltigen Knotchen durch Einkapselung von Cholesterintafeln. Zieglers Beitr 13: 76, 1983

5) Waltner JC, Karatay S: Cysts of the mastoid bone. Arch Otolaryngol 46: 398-404, 1947

6) Graham MD, Kemink JL, Latack JT, Kartush JM: The giant cholesterol cyst of the petrous apex: a distinct clinical entity. Laryngoscope 95: 1401-1406, 1985

7) Manasse P: Uber Granulationsgeschwulste mit Fremdkorperriesenzellen. Virchows Arch 136: 245, 1894

8) Griffith AJ, Terrell JE: Transsphenoid endoscopic management of petrous apex cholesterol granuloma. Otolaryngol Head Neck Surg 114: 91-94, 1996

9) Samadian M, Vazirnezami M, Moqaddasi H, Rakhshan M, Khormaee F, Ashraf H: Endoscopic transrostraltranssphenoidal approach to petrous apex cholesterol granuloma: case report. Turk Neurosurg 19: 106-111, 2009
10) Georgalas C, Kania R, Guichard JP, Sauvaget E, Tran Ba Huy P, Herman P: Endoscopic transsphenoidal surgery for cholesterol granulomas involving the petrous apex. Clin Otolaryngol 33: 38-42, 2008

11) Fucci MJ, Alford EL, Lowry LD, Keane WM, Sataloff RT: Endoscopic management of a giant cholesterol cyst of the petrous apex. Skull Base Surg 4: 52-58, 1994

12) Montgomery WW: Cystic lesions of the petrous apex: transsphenoid approach. Ann Otol Rhinol Laryngol 86(4 Part 1): 429-435, 1977

13) Oyama K, Ikezono T, Tahara S, Shindo S, Kitamura T, Teramoto A: Petrous apex cholesterol granuloma treated via the endoscopic transsphenoidal approach. Acta Neurochir (Wien) 149: 299-302; discussion 302, 2007

14) Sabin HI, Bordi LT, Symon L: Epidermoid cysts and cholesterol granulomas centered on the posterior fossa: twenty years of diagnosis and management. Neurosurgery 21: 798-805, 1987

15) DiNardo LJ, Pippin GW, Sismanis A: Image-guided endoscopic transsphenoidal drainage of select petrous apex cholesterol granulomas. Otol Neurotol 24: 939-941, 2003

16) Michaelson PG, Cable BB, Mair EA: Image-guided transphenoidal drainage of a cholesterol granuloma of the petrous apex in a child. Int J Pediatr Otorhinolaryngol 57: 165-169, 2001

17) Kusumi M, Fukushima T, Mehta AI, Cunningham CD 3rd, Friedman AH, Fujii K: Middle fossa approach for total resection of petrous apex cholesterol granulomas: use of vascularized galeofascial flap preventing recurrence. Neurosurgery 72(1 Suppl Operative): 77-86; discussion 86, 2013

18) Paluzzi A, Gardner P, Fernandez-Miranda JC, Pinheiro-Neto CD, Scopel TF, Koutourousiou M, Snyderman CH: Endoscopic endonasal approach to cholesterol granulomas of the petrous apex: a series of 17 patients: clinical article. J Neurosurg 116: 792-798, 2012

19) Jaberoo MC, Hassan A, Pulido MA, Saleh HA: Endoscopic endonasal approaches to management of cholesterol granuloma of the petrous apex. Skull Base 20: 375-379, 2010

Address reprint requests to: Shunsuke Shibao, MD, Department of Neurosurgery, Keio University School of Medicine, 35 Shinano-machi, Shinjuku-ku, Tokyo 160-8582, Japan.

e-mail: pochisuke616@mac.com 